J. Sustain. Wireless Syst.

Vol.02/ No. 2

Pages: 68- 75

http://irojournals.com/irosws/

DOI: https://doi.org/10.36548/jsws.2020.2.002

\title{
Machine Learning Implementation in Cognitive Radio Networks with Game-Theory Technique
}

\author{
Dr. Jennifer S. Raj, \\ Department of ECE, \\ Gnanamani College of Technology, \\ Namakkal, India. \\ Email: jennifer.raj@gmail.com.
}

\begin{abstract}
Significant enhancement of spectrum utilization can be performed by means of Cognitive Radio technology. A game theory based Cognitive Radio Network with Dynamic Spectrum Allocation model is proposed in this paper. $\mathrm{M}|\mathrm{M}| 1$ queuing model is implemented along with Preemptive Resume Priority for accommodation of all the cases. An Incremental Weights-Decremental Ratios (IW-DR) algorithm based on priority-based scheduling is used for supplementing this theory. Regression models are used for restructuring and improving the efficiency of the system.
\end{abstract}

Keywords: Regression; Game Theory; Queuing Theory; Spectrum Allocation; Cognitive Networks;

\section{Introduction}

The developments in wireless spectrum and growing demands has an issue of a serious insufficiency in the unlicensed spectrum in this rapidly improving digital age [1]. The number of devices and applications dependant exclusively on the unlicensed bands availability is increasing exponentially. Under-utilization of licensed bands and congestion in unlicensed bands are the contrary scenarios in these devices and applications. The use of the channels effectively is performed by the wireless services prospective growth [2]. The limited radio spectrum and its deficiency issues can be alleviated by the novel solution of Cognitive Radio (CR) Networks. Depending on the spectrum environment, the configurations are adjusted for their competence with the devices that are intelligent and spectrum-agile in the CR Networks [3]. Primary and Secondary Users (PU \& SU) are the two major types of CR networks that use the spectrum of obligatory licensed users and licensed spectrum that is unused with opportunistic access respectively. Dynamic Spectrum Access is the composite term used for this feature. Avoiding the Quality of Service loss and harmonious coexistence are ensured in the corresponding networks on a peaceful basis [4].

The dynamic spectrum allocation is proposed in leasing, auctions, game theory and other such applications. The contradictory interest of the players and their interactions are modelled and developed using robust tool in the game theory [5]. Rationality of every player in the game is a premise in implementing the game theory. The payoffs of the players can be maximized individually by the SU by the rational game players. The channel allotment and payoff is as follows [6]. The PUs are relinquished by the available channels for competing with the SU players in the dynamic non-cooperative game as proposed in this work. The SU collision is dodged in a fashion that the available networks stay or switch 
J. Sustain. Wireless Syst.

Vol.02/ No. 2

Pages: 68- 75

http://irojournals.com/irosws/

DOI: https://doi.org/10.36548/jsws.2020.2.002

between each other in this strategy [7]. On acquiring the accessible channel in the SU on achieving the equilibrium point of the game. A dynamic spectrum access technique is set up in the game-theory environment. Formulation of Nash equilibrium is performed for the game [8]. The simulation of the game is performed and implemented using regression techniques. Based on the channel availability, the traffic surpasses system failure. This is a major setback of this system. This setback is amended by the work in this paper. The IW-DR Scheduling Algorithm and M|M|1 Queuing Networks with PRP is used for resolving this issue and relieving the congestion [9].

The performance of all SUs in spectrum allocation of Game theory cannot be guaranteed to be of the same levels. The Incremental Weights-Decremental Ratios (IW-DR) is the scheduling algorithm that is customized for this purpose in this work. According to the sensitivity order and type of application, QoS is achieved by the application of secondary user that is delay-sensitive for the bolster [10]. The continuous datasets are analyzed with several regression algorithms for implementation of learning in the game. For this purpose, Random Forest Regression, Decision Tree Regression, Support Vector Regression, Polynomial Regression and Simple Linear Regression is used for prediction of optimal probability. The channels available $\mathrm{N}_{\mathrm{C}}$ and network count $\mathrm{N}_{\mathrm{N}}$ for an optimal probability is obtained using the game environment that is simulated [11].

\section{Architecture of the System}

In $X_{r}$ channels, the competing SUs or the total network count is represented by $Y_{r}$ and at any particular time instance, the channel count available is represented by $X_{r}$ in a dynamically changing game environment for the experimental scenario. Assume $\left(1 / \lambda_{\mathrm{T}}\right)$ be the arrival time and $\mathrm{T}_{\mathrm{EQ}}$ is the channel accommodation time which is lesser than the arrival time. Two subcases namely switch and stay are categorized based on $\mathrm{Y}_{\mathrm{r}}$ and $\mathrm{X}_{\mathrm{r}}$. Figure 1 represents the proposed system in which the channels are represented by squares. A 10 channel 8 network system is used for this purpose. In step 1, (SU5, SU6), (SU2, SU4) and (SU3, SU1, SU7) tuples have a collision. Similarly, in step 2, (SU4, SU6) and (SU2, SU1) as well as in step 3, (SU4, SU1) tuples have collision. Based on the optimal probability, the problem in each scenario is dealt differently.

A game is simulated using Game Algorithm replicating the game scenario. Further, the time taken for achieving equilibrium is calculated for every value of $\mathrm{P}$ that ranges between 0.99 and 0.01 .100 iterations of this process is performed for every tuple of $(\mathrm{M}, \mathrm{N}$ and $\mathrm{P})$ and the calculation of the mean equilibrium time is done. The least equilibrium time is selected for optimization of probability for the $(\mathrm{M}, \mathrm{N})$ tuple. This input attains an output of $\left(\mathrm{M}, \mathrm{N}, \mathrm{P}_{0}\right)$ tuple. 
J. Sustain. Wireless Syst.

Vol.02/ No. 2

Pages: 68- 75

http://irojournals.com/irosws/

DOI: https://doi.org/10.36548/jsws.2020.2.002

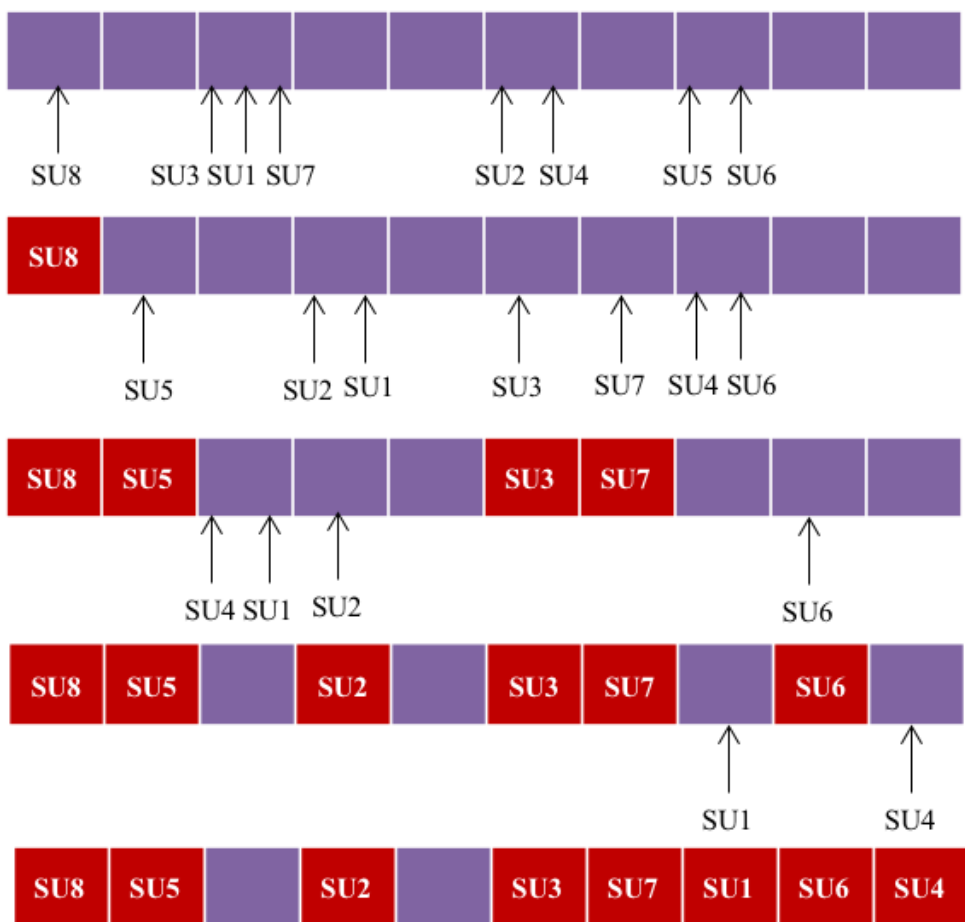

Figure 1: 10 channel-8 network dynamic game illustration

\section{Proposed Work}

The system in which just one independent variable is available is termed as simple linear regression and the availability of multiple independent variables is termed as multiple linear regression [12]. The mathematical representation of this regression method is as follows:

$$
\begin{array}{r}
f(x)=\omega_{0}+\omega_{1} x_{1}+\omega_{2} x_{2}+\cdots+\omega_{d} x_{d} \\
f(x)=\omega_{0}+\sum_{j=1}^{d} \omega_{i} k_{i}(x)----(2)
\end{array}
$$

The input variables $\mathrm{x}=\left(\mathrm{x}_{1}, \mathrm{x}_{2}, \ldots \mathrm{x}_{\mathrm{d}}\right)$ and linear parameter functions $\omega=\left(\omega_{0}, \omega_{1}, \ldots \omega_{\mathrm{d}}\right)$ are the reason behind the name linear regression. Any permanent offset in the information is allowed by a parameter $\omega_{0}$.

In comparison to Linear Regression, a more versatile algorithm called Polynomial Regression is available which is quite similar in certain aspects [13]. Basis function is the major difference in these algorithms and is represented by the following expression: 
J. Sustain. Wireless Syst.

Vol.02/ No. 2

Pages: 68- 75

http://irojournals.com/irosws/

DOI: https://doi.org/10.36548/jsws.2020.2.002

$$
\varphi_{i}(x)=x^{i}----(3)
$$

(M-1) is the degree of polynomial. Constant, first order and second order polynomials are chosen based on the value of $\mathrm{M}$ which can be 0,1 or 2 respectively. The training dataset that fits our application best is chosen.

The classification principle used for SVM is also used in Support Vector Regression [14]. The hyperplane and data points are separated maximally by the multidimensional space where data is separated using the hyperplane. The limits are maintained within $\varepsilon$ for deviation so as to have the allowed tolerance of errors and to calculate the value of $\mathrm{f}(\mathrm{x})$. The linear function can be calculated with the following expression:

$$
f(x)=\omega x+\beta----(4)
$$

The branches that represent the item observation is used for concluding regarding the leaves that represent the target value of the item that provides the predictive model of the decision tree using the decision tree regression [15]. Classification and Regression Trees (CART) is used for building the decision tree using the metric called Gini Index in which every class probability is represented by $\rho_{\mathrm{I}}$ and class by $\mathrm{C}$. This is represented by the following expression:

$$
\text { Gini Index }=1-\sum_{i=1}^{c}\left(\rho_{i}\right)^{2}
$$

The mean of individual trees are predicted and presented as output and the training time is used for construction of the decision trees in a large number for regression in ensemble learning technique using random decision forest. The decision tree regressions over fitting problem is solved using this method. The decision tree feature can be calculated using the following expression where the random forest model trees are calculated using the major feature RFfij:

$$
\text { Norm } f i_{m}=\frac{f i_{m}}{\sum_{n \text { call features }} f i_{n}}------(6)
$$

\section{Results and Discussion}

Scientific Python Development Environment (SPYDER) in Python is used along with Anaconda framework as an integrated development environment (IDE) which is open source for the purpose of simulation. Reasoning and learning capabilities are to be equipped in the cognitive radio networks to 
J. Sustain. Wireless Syst.

Vol.02/ No. 2

Pages: 68- 75

http://irojournals.com/irosws/

DOI: https://doi.org/10.36548/jsws.2020.2.002

enable the system to operate efficiently. The need for self-learning is the inability of the channels to search and sense for other channels in the surroundings. An efficient dataset is used for coupling learning along with the games. The channels available $\mathrm{N}_{\mathrm{C}}$ and network count $\mathrm{N}_{\mathrm{N}}$ for an optimal probability is obtained using the game environment that is simulated.

Table 1: Probability comparison for various strategies

\section{EXP RFR DTR SVR PR SLR}

\begin{tabular}{|l|l|l|l|l|l|l|}
\hline 50 & 0.54 & 0.56 & 0.54 & 0.55 & 0.49 & 0.58 \\
\hline 45 & 0.55 & 0.55 & 0.54 & 0.55 & 0.58 & 0.61 \\
\hline 40 & 0.66 & 0.66 & 0.68 & 0.64 & 0.66 & 0.64 \\
\hline 35 & 0.67 & 0.67 & 0.68 & 0.71 & 0.69 & 0.67 \\
\hline 30 & 0.71 & 0.73 & 0.73 & 0.73 & 0.71 & 0.70 \\
\hline 25 & 0.70 & 0.74 & 0.73 & 0.73 & 0.72 & 0.73 \\
\hline 30 & 0.72 & 0.72 & 0.73 & 0.72 & 0.74 & 0.76 \\
\hline 15 & 0.77 & 0.77 & 0.77 & 0.74 & 0.76 & 0.79 \\
\hline 10 & 0.83 & 0.81 & 0.81 & 0.81 & 0.82 & 0.82 \\
\hline
\end{tabular}

The predictive algorithms used here are Elastic Net Regression, Support Vector Regression and Linear Regression. Random Forest Regression, Decision Tree Regression, Support Vector Regression, Polynomial Regression and Simple Linear Regression are also implemented in this work as represented in Table 1. 99.8\% enhancement is obtained by the results of Simple Vector Regression and $97.7 \%$ for Linear Regression. The available channel count is surpassed by the SUs traffic which did not support the case. The IW-DR Scheduling Algorithm and M|M|1 Queuing Networks with PRP is used for resolving this issue.

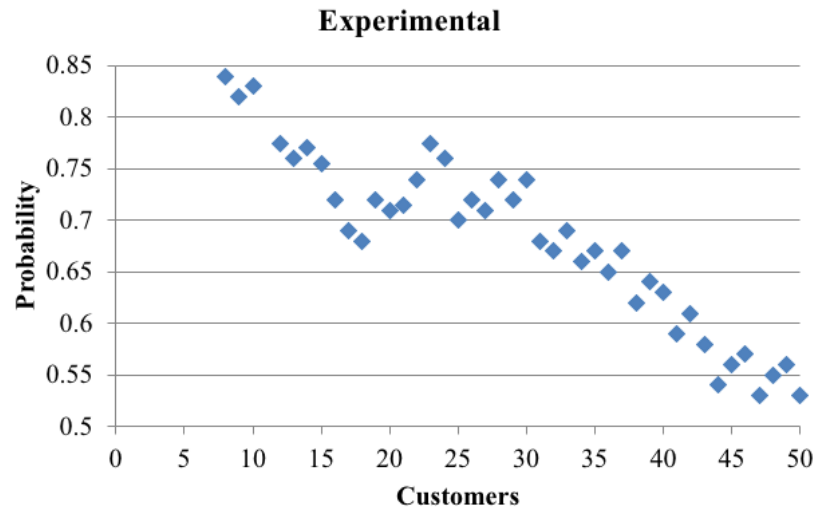


J. Sustain. Wireless Syst.

Vol.02/ No. 2

Pages: 68- 75

http://irojournals.com/irosws/

DOI: https://doi.org/10.36548/jsws.2020.2.002

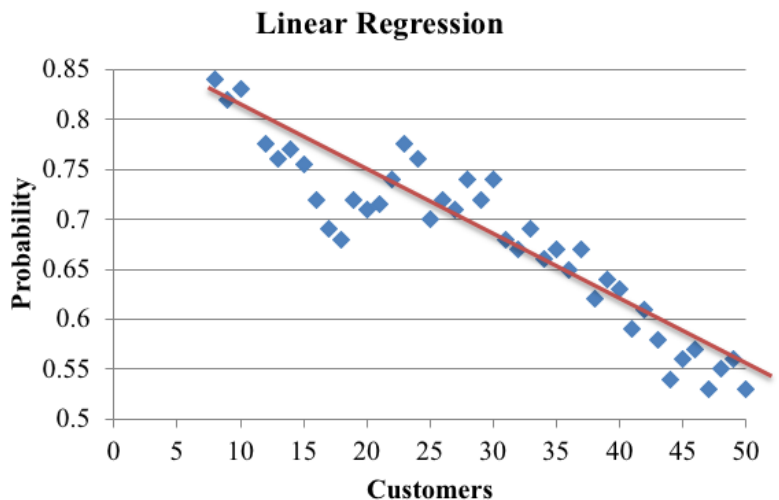

(b)

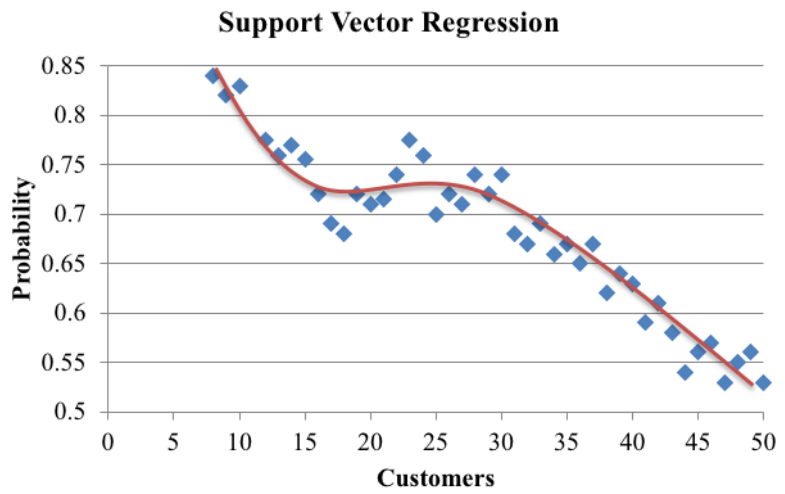

(d) (a)

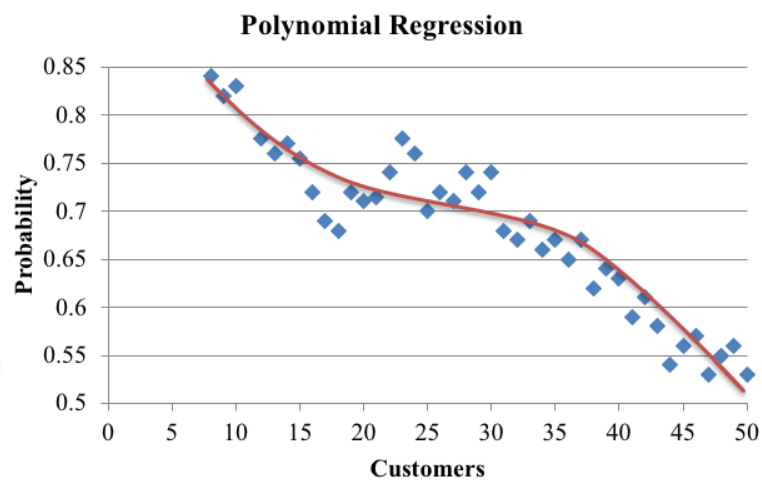

(c)

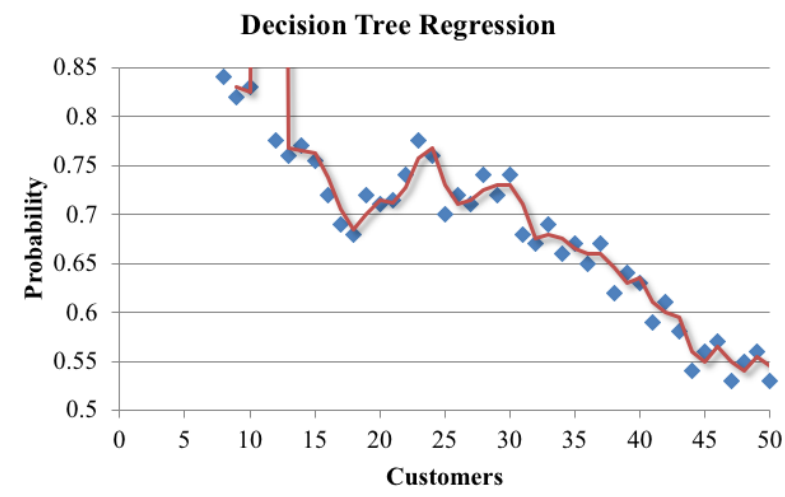

(e)

Figure 2: Comparison of Optimal Probability Vs Customers in (a)Experiment, (b) Linear Regression,

(c) Polynomial Regression, (d)Support Vector Regression and (e) Decision Tree Regression

The total datasets simulated here is 820 . They range between 10 to 50 for several possible combinations of game algorithms. Out of these datasets, for testing purpose, we use 164 datasets and for training purpose we use 656 datasets. A 3 column database is used for storing the database - namely Optimal Switching Probability $\left(\mathrm{P}_{0}\right)$, Number of Networks $(\mathrm{N})$ and Number of Channels $(\mathrm{M})$. Five regression algorithms namely Random Forest Regression, Decision Tree Regression, Support Vector Regression, Polynomial Regression and Simple Linear Regression are proposed in this work. The experimental results obtained by the Game algorithm for comparison of optimal probability and the customers is represented in Figure 2 (a). Comparison with the other compared algorithms is shown in Figure 2 (b), (c), (d) and (e). With the raise in the number of competitors, the switching probability drops according to the graph.

\section{Conclusion}

Dynamic Spectrum Allocation is improved by the implementation of the non-cooperative dynamic game. $\mathrm{M}|\mathrm{M}| 1$ Queuing Network with PRP is used for steering the secondary user traffic in this paper. 
J. Sustain. Wireless Syst.

Vol.02/ No. 2

Pages: 68- 75

http://irojournals.com/irosws/

DOI: https://doi.org/10.36548/jsws.2020.2.002

The users of various classes in the queues are provided equality using the IW-DR Scheduling Algorithm. Five regression algorithms namely Random Forest Regression, Decision Tree Regression, Support Vector Regression, Polynomial Regression and Simple Linear Regression are compared and analyzed with the results of the proposed work for inducing the non-cooperative game with self-learning capability.

\section{References}

[1] Haoxiang, W. (2019). MULTI-OBJECTIVE OPTIMIZATION ALGORITHM FOR POWER MANAGEMENT IN COGNITIVE RADIO NETWORKS. Journal of Ubiquitous Computing and Communication Technologies (UCCT), 1(02), 97-109.

[2] Darney, P. E., \& Jacob, I. J. (2019). PERFORMANCE ENHANCEMENTS OF COGNITIVE RADIO NETWORKS USING THE IMPROVED FUZZY LOGIC. Journal of Soft Computing Paradigm (JSCP), 1(02), 57-68.

[3] Valanarasu, M. R., \& Christy, A. (2019). COMPREHENSIVE SURVEY OF WIRELESS COGNITIVE AND 5G NETWORKS. Journal of Ubiquitous Computing and Communication Technologies (UCCT), 23-32.

[4] Pandian, M. D. (2019). ENHANCED NETWORK PERFORMANCE AND MOBILITY MANAGEMENT OF IOT MULTI NETWORKS. Journal of trends in Computer Science and Smart technology (TCSST), 1(02), 95-105.

[5] Raj, J. S., \& Smys, S. (2019). VIRTUAL STRUCTURE FOR SUSTAINABLE WIRELESS NETWORKS IN CLOUD SERVICES AND ENTERPRISE INFORMATION SYSTEM. Journal of ISMAC, 1(03), 188-205.

[6] Isaac, M. (2017). Machine learning prediction algorithm to determine best performing routes in cognitive radio networks (Doctoral dissertation, Uganda Technology And Management University).

[7] Mangairkarasi, S., Sarankapani, R., \& Arivudainambi, D. (2020). A Game-Theoretic Approach for Cognitive Radio Networks using Machine Learning Techniques (No. 2313). EasyChair.

[8] Bharathi, S., Kumar, D., \& Ram, D. (2018). Defence against responsive and non-responsive jamming attack in cognitive radio networks: an evolutionary game theoretical approach. The Journal of Engineering, 2018(2), 68-75.

[9] Qadir, J. (2016). Artificial intelligence based cognitive routing for cognitive radio networks. Artificial Intelligence Review, 45(1), 25-96.

[10] Jaishanthi, B., Ganesh, E. N., \& Sheela, D. (2019). Priority-based reserved spectrum allocation by multi-agent through reinforcement learning in cognitive radio network. Automatika, 60(5), 564-569.

[11] Kwasinski, A., Wang, W., \& Mohammadi, F. S. (2020). Reinforcement Learning for Resource Allocation in Cognitive Radio Networks. Machine Learning for Future Wireless Communications, 27-44. 
J. Sustain. Wireless Syst.

Vol.02/ No. 2

Pages: 68- 75

http://irojournals.com/irosws/

DOI: https://doi.org/10.36548/jsws.2020.2.002

[12] Gupta, R., \& Gupta, P. C. (2017). COGNITIVE RADIO NETWORKS IMPLEMENTATION FOR SPECTRUM UTILIZATION IN HADOTI (RAJASTHAN) REGION. VINDHYA BHARTI.

[13] Umbert, A., Sallent, O., Pérez-Romero, J., Sánchez-González, J., Collins, D., \& Kist, M. (2018, May). An experimental assessment of channel selection in cognitive radio networks. In IFIP International Conference on Artificial Intelligence Applications and Innovations (pp. 78-88). Springer, Cham.

[14] Tuberquia, M., \& Hernandez, C. (2018). New Approaches in cognitive radios using evolutionary algorithms. International Journal of Electrical and Computer Engineering, 8(3), 1636.

[15] Roumeliotis, A. J., Poulakis, M. I., Vassaki, S., \& Panagopoulos, A. D. (2017). Radio Resources Management Optimization in Cognitive Radio Networks. New Directions in Wireless Communications Systems: From Mobile to 5G, 433.

\section{Author's Biography}

Dr. Jennifer S Raj received the Ph.D degree from Anna University and Master's Degree in communication System from SRM University, India. Currently she is working in the Department of ECE, Gnanamani College of Technology, Namakkal, India. She is a life member of ISTE, India. She has been serving as Organizing Chair and Program Chair of several International conferences, and in the Program Committees of several International conferences. She is book reviewer for Tata Mc Graw hill publication and publishes more than fifty research articles in the journals and IEEE conferences. Her interests are in wireless Health care informatics and body area sensor networks 